\title{
Variable delay using stationary and localized Brillouin dynamic gratings
}

\author{
Yair Antman ${ }^{\mathrm{a}}$, Nikolay Primerov ${ }^{\mathrm{b}}$, Juan Sancho ${ }^{\mathrm{b}}$, Luc Thevenaz ${ }^{\mathrm{b}}$, and Avi Zadok ${ }^{* \mathrm{a}}$ \\ ${ }^{a}$ Faculty of Engineering, Bar-Ilan University, Ramat-Gan 52900 Israel; \\ ${ }^{\mathrm{b}}$ Ecole Polytechnique Fédérale de Lausanne, Institute of Electrical Engineering, SCI-STI-LT Station \\ 11, 1015 Lausanne, Switzerland; \\ ${ }^{\S}$ Permanent address: iTEAM Institute, Universidad Politécnica de Valencia, 46022 Valencia, Spain.
}

\begin{abstract}
Reflections from movable, dynamic acoustic gratings in polarization maintaining (PM) fibers are employed in the long variable delay of periodic, isolated pulses. The gratings are introduced by stimulated Brillouin scattering (SBS) interaction between two counter-propagating pump waves, which are spectrally detuned by the Brillouin frequency shift of the PM fiber and are both polarized along one of its principal axes. The gratings are interrogated by the reflections of read-out signals that are polarized along the orthogonal principal axis. High-rate phase modulation of both pump waves by a pseudo-random binary sequence introduces dynamic gratings that are both localized and stationary, at specific locations in which the modulated pumps are correlated. The separation between adjacent correlation peaks can be made arbitrarily long. Long variable delays are readily obtained by scanning the grating along the fiber, via changing either the length or the rate of the modulation sequence. At the same time, the short length of the gratings, on the order of a $\mathrm{cm}$, accommodates the delay of broadband pulses. The technique is therefore free of the delay-times-bandwidth product limitation that undermines the performance of SBS-based 'slow light' delay: we report the delay 1-ns long pulses by as much as $770 \mathrm{~ns}$. In addition, the combined reflections from two dynamic gratings with a variable separation are used to implement radio-frequency photonic filters of tunable free spectral range. At the current stage, the technique is restricted by noise from residual scattering that takes place outside of the correlation peaks. Hence, it is thus far limited to the processing of repetitive signals, for which the noise may be effectively averaged out.
\end{abstract}

Keywords: Variable group delay, stimulated Brillouin scattering, slow and fast light, nonlinear fiber optics, dynamic acoustic gratings

\section{INTRODUCTION}

Stimulated Brillouin scattering (SBS) is driven by the interference between two counter-propagating optical waves that are detuned in frequency. The intensity of the two waves added together includes a slowly-traveling beating term, whose frequency $\Omega$ equals the difference between the frequencies of the two optical waves. The intensity wave introduces traveling variations in the fiber material density, or an acoustic wave, through electrostriction [1]. The acoustic wave is accompanied by a traveling grating of refractive index variations, due to the photo-elastic effect [1]. The traveling grating can induce a coupling between the two optical waves. Effective coupling requires that the frequency difference $\Omega$ closely matches the Brillouin frequency shift $\Omega_{B}$ of the fiber [1], which is $\sim 2 \pi \cdot 11 \mathrm{GHz}$ in standard fibers at $1550 \mathrm{~nm}$ wavelength.

Over the last three years, SBS-based dynamic acoustic gratings over polarization maintaining (PM) fibers have been drawing increasing attention [2-14]. Dynamic gratings are introduced by two counter-propagating pump waves, separated in frequency by $\Omega_{B}$ and co-polarized along one principal axis of the PM fiber. The SBS interaction between the two pumps introduces a grating of refractive index variations, which can be switched on and off and even moved along the fiber, depending on the profiles of the pump waves. The dynamic grating is interrogated by a third, probe signal, which is polarized along the orthogonal principal axis of the PM fiber. Due to the relatively large birefringence of such fibers, the frequency of the reading signal must be detuned from those of the pumps, typically by a few tens of GHz [2].

*Avinoam.Zadok@biu.ac.il Tel.: +972-3-5318882; Fax: +972-3-7384051.

Advances in Slow and Fast Light V, edited by Selim M. Shahriar, Frank A. Narducci,

Proc. of SPIE Vol. 8273, 82730C - (c) 2012 SPIE · CCC code: 0277-786X/12/\$18 · doi: 10.1117/12.914725 
The reading signal is back-reflected by the dynamic grating into a fourth wave, which can be measured to monitor the entire process. In this work we focus on the application of dynamic gratings may as 'movable mirrors', which represent a new potential platform for all-optical variable group delay [7, 14]. Dynamic gratings-based delay could be free of the delay-bandwidth product limitations that undermine the performance of so-called SBS slow-light systems [15-19]. However, the reflection of broadband interrogating waveforms requires dynamic gratings that are localized and short in length.

When two continuous wave (CW) pumps are used, a uniform dynamic grating is introduced along the entire PM fiber, leading to narrow-band reflectivity spectrum. Localized dynamic gratings have been generated using pump pulses [8, 14]. However, the generation of short gratings is restricted by the relatively long acoustic lifetime $\tau \sim 6 \mathrm{~ns}$. In addition, the gratings must be 'refreshed' by periodic pump pulses every $\tau$, hence the strength of reflection is temporally varying. Localized gratings may be generated using Brillouin optical correlation-domain analysis (B-OCDA) techniques, in which the instantaneous frequencies of constant-magnitude pump and probe waves, that are nominally detuned by $\Omega$, are synchronously modulated by a common sine wave $[20,21]$. Due to the modulation, the frequency difference between the two counter-propagating waves remains stationary at particular fiber locations only, known as correlation peaks, whereas the frequency difference elsewhere is oscillating [20,21]. Consequently, effective SBS amplification is restricted to the correlation peaks, and probe power measurements may convey localized information. The unambiguous measurement range of B-OCDA, however, is restricted to the separation between periodic correlation peaks, which is typically several hundred times the spatial resolution. Hence when simple sine-wave frequency modulation is used, tight trade-offs prevail between the B-OCDA range and resolution [20, 21].

In this work, we propose, demonstrate and apply a novel technique for the generation of SBS dynamic gratings that are both localized and stationary. The method relies on the phase modulation of the two pump waves by a common pseudorandom bit sequence (PRBS), with a symbol duration $T$ that is much shorter than $\tau$. The method allows for an effective SBS interaction in discrete, cm-scale correlation peaks only. The separation between neighboring correlation peaks is governed by the length of the PRBS, and it is uncorrelated with the length of the localized gratings. We experimentally demonstrate the generation of cm-scale, stationary and localized dynamic gratings over PM fibers of up to $100 \mathrm{~m}$ in length [22]. The gratings are applied to the variable delay of periodic, $1 \mathrm{~ns}-$ long pulses by up to $770 \mathrm{~ns}$. In addition, the combined reflections from a pair of dynamic gratings are used to implement a tow-tap, radio-frequency (RF) photonic filter with a variable free spectral range (FSR). The results illustrate the potential impact of the proposed technique on various applications of dynamic gratings. The method is equally applicable to the generation of a localized SBS interaction over standard fibers.

The remainder of this paper is organized as follows. The principle of operation of PRBS-driven, localized and stationary dynamic gratings is explained in section 2, alongside numerical simulations of the spatio-temporal grating profile. The experimental setup is described in section 3. Experimental results of variable delay and RF filtering are provided in sections 4 and 5, respectively. Concluding remarks are given in section 6 .

\section{PRINCIPLE OF OPERATION}

Let us denote the optical fields of the two pump waves of an SBS dynamic grating as $E_{1,2}(t, z)$, respectively, where $t$ stands for time and $z$ represents position along a PM fiber of length $L$. Pump $E_{1}$ enters the fiber at $z=0$ and propagates along the positive $z$ direction, whereas pump $E_{2}$ propagates from $z=L$ in the negative $z$ direction. The optical frequencies of the two pump waves, $\omega_{1,2}$, are separated by $\Omega \sim \Omega_{B}$. The complex envelopes of the two waves are denoted

by $A_{1,2}(t, z)$, so that:

$$
\begin{gathered}
E_{1}(t, z)=A_{1}(t, z) \exp \left(j \omega_{1} t\right)+c . c \\
E_{2}(t, z)=A_{2}(t, z) \exp \left[j\left(\omega_{1}+\Omega\right) t\right]+c . c .
\end{gathered}
$$

In our proposed scheme for generating stationary and localized gratings, the phases of both pump waves are modulated by a common PRBS with symbol duration $T$ : 


$$
A_{1}(t, z=0)=A_{2}(t, z=L)=A(t)=A_{0}\left\{\sum_{n} \operatorname{rect}[(t-n T) / T] \exp \left(j \varphi_{n}\right)\right\}
$$

In Eq. (3), $\varphi_{n}$ is a random phase variable which equals either 0 or $\pi$, rect $(\xi)$ equals 1 for $|\xi|<0.5$ and zero elsewhere, and $A_{0}$ denotes the constant magnitude of both pump waves. In the following, we consider both pumps to be of the same power, for simplicity. The analysis is equally valid for pumps having different power levels. The modulation is synchronized so that the phases of the two pumps, at their respective entry points into the fiber, are equal for all $t$. Both pumps are polarized along the same principal axis of the PM fiber, denoted as $\hat{\mathbf{x}}$.

Consider next the magnitude of the acoustic density wave $Q(t, z)$ of frequency $\Omega$ that is generated by the two pumps. The driving force for the acoustic wave is proportional to the product $A_{1}(t, z) A_{2}^{*}(t, z)[1,23]$. Subject to the pumps modulation in the form of Eq. (3), the driving force scales with $A\left(t-z / v_{g}\right) A^{*}\left[t-z / v_{g}-\theta(z)\right]$, where $\theta(z) \equiv(2 z-L) / v_{g}$ is a position-dependent temporal offset and $v_{g}$ the group velocity of light in the fiber.

We can distinguish between the temporal evolutions of $Q(t, z)$ in two regions. Within a short section surrounding the center of the fiber at $z=L / 2, \theta(z) \approx 0$ and $A\left(t^{\prime}-z / v_{g}\right) A^{*}\left[t^{\prime}-z / v_{g}-\theta(z)\right]=\left|A_{0}\right|^{2}$. The two modulated pump waves are correlated in this particular location, hence the driving force for the acoustic grating generation is stationary and of constant phase. Consequently, the acoustic grating in the vicinity of $z=L / 2$ is allowed to build up to its steady state magnitude. The width of the correlation peak is on the order of $\Delta z=\frac{1}{2} v_{g} T$, corresponding to the spatial extension of a symbol of the PRBS. In all other $z$ locations, on the other hand, the product $A\left(t-z / v_{g}\right) A^{*}\left[t-z / v_{g}-\theta(z)\right]$ is randomly alternating between $\pm\left|A_{0}\right|^{2}$ due to the random phase change between 0 and $\pi$, on every symbol duration $T<<\tau$. Over the lifetime $\tau$, the integrated driving force for the acoustic field generation averages to a zero expectation value and the buildup of $Q(t, z)$ outside the correlation peak is inhibited. The acoustic grating that is generated by PRBS modulation of the pump waves is therefore both stationary and localized. (Note that the strength of the grating outside the correlation peak, however weaker, has a non-zero standard deviation and time varying amplitude.)

The separation $Z$ between adjacent peaks equals $\frac{1}{2} M v_{g} T$, where $M$ is the PRBS length. The correlation peaks, with the exception of the zeroth-order one, can be scanned across a fiber under test through changing the code length factor $M$ or the symbol duration $T$. The unambiguous measurement range can be made arbitrarily long with increasing $M$, while retaining the above resolution of $\Delta z$. Since there is no frequency modulation of the pumps the spatial period of the acoustic grating does not oscillate, and the subsequent readout of the acoustic grating by a $\hat{\mathbf{y}}$ polarized interrogating wave becomes considerably simpler than in B-OCDA over PM fibers [6]. The technique is not restricted to dynamic gratings over PM fibers: it is equally applicable to the modulation of pump and probe waves interacting along a standard fiber.

Figure 1 shows simulation results of $Q(t, z)$ within a $1 \mathrm{~m}$ long fiber, subject to PRBS phase modulation of both pump waves with $T=200 \mathrm{ps}$. The frequency shift $\Omega$ between the two modulated pumps was chosen to match $\Omega_{B}$ of the fiber. The simulation predicts the build-up of a localized and stationary grating in a narrow region at the center of fiber, as suggested by the above considerations. The experimental realization of such gratings is presented next. 


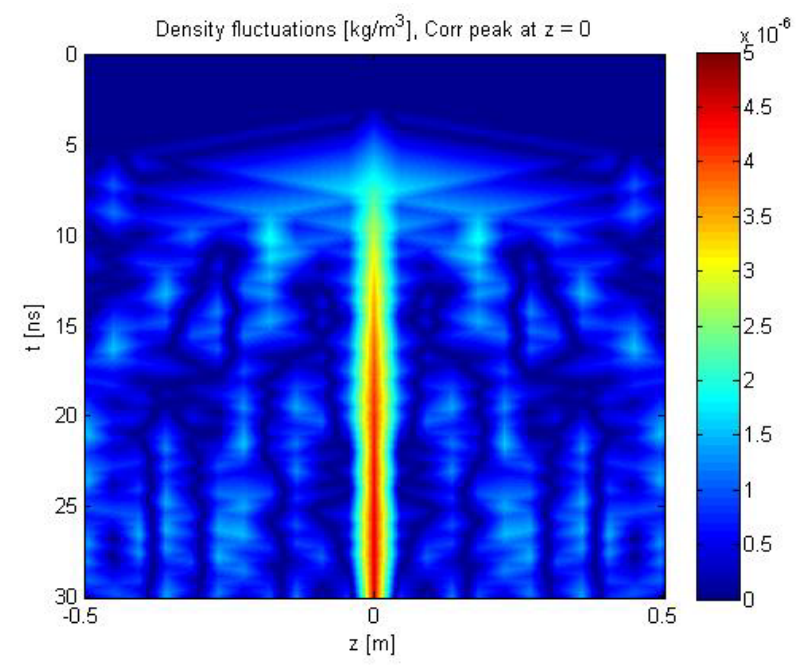

Fig. 1: Simulated magnitude of the acoustic wave density fluctuations, (in $\mathrm{kg} / \mathrm{m} 3$ ), that is generated by two pumps, which are phase-modulated by a common pseudo-random bit sequence, as a function of position $z$ and time $t$ within a $1 \mathrm{~m}$-long fiber. The frequency separation between the two pumps was chosen to match the Brillouin frequency shift in the fiber. The modulation symbol rate $T$ was $200 \mathrm{ps}$.

\section{EXPERIMENTAL SETUP}

Figure 2 illustrates the experimental setup that was used for the generation and characterization of dynamic acoustic gratings, driven by random phase modulation of both pump waves. A single distributed feed-back (DFB) laser diode of frequency $\omega_{1}$ was used to generate both pump waves. The output of the pumps DFB was modulated by an electro-optic phase modulator (EOM), which was driven by a PRBS generator. The output peak-to-peak voltage of the PRBS generator was adjusted to match $V_{\pi}$ of the $\operatorname{EOM}(\sim 3.7 \mathrm{~V})$, and its clock rate was controlled by a microwave generator. The modulated DFB light was split in two arms. Light in one path was amplified by an erbium-doped fiber amplifier (EDFA) to $\left|A_{1}\right|^{2} \sim 200 \mathrm{~mW}$, polarized along the $\hat{\mathbf{x}}$ principal axis of a PM fiber, and launched into a section of specialty PM fiber under test (FUT) as pump 1. Light in the other arm was modulated by a second EOM, which was driven by a sine wave of frequency $\Omega \sim 2 \pi \cdot 10 \mathrm{GHz}$ from a second microwave generator. The EOM in this arm was biased to suppress the optical carrier at $\omega_{1}$. The upper modulation sideband of frequency $\omega_{2}=\omega_{1}+\Omega$ was selected by a narrowband fiber Bragg grating (FBG), amplified by a second EDFA to $\left|A_{2}\right|^{2} \sim 200 \mathrm{~mW}$, and launched along the $\hat{\mathbf{x}}$ axis from the opposite end of the FUT as pump 2. The residual carrier wave and the other modulation sideband of pump 2 were rejected by the FBG.

Light from a second DFB laser was used to generate the readout signal wave. The optical frequency of the signal DFB was adjusted via temperature and current control to match the frequency of maximum reflectivity of the acoustic dynamic grating along the $\hat{\mathbf{y}}$ principal axis [2]:

$$
\omega_{\text {sig }}=\omega_{2}+\Delta \omega=\omega_{2}+\frac{\Delta n_{g}}{n} \omega_{2}
$$

In Eq. (4), $\Delta n_{g}<<1$ is the difference between the group indices of propagation along $\hat{\mathbf{x}}$ and $\hat{\mathbf{y}}$ axes, normally nearly identical to the phase birefringence $\Delta n$ in standard PM fibers, and $n$ denotes the mean value of the two indices [2]. Some of the measurements relied on continuous wave readout signals, whereas in other experiments a third EOM was used to generate readout pulses or sine-wave modulation, as necessary. The readout signal wave was polarized along the $\hat{\mathbf{y}}$ principal axis of the PM FUT, and was launched to probe the acoustic dynamic grating. The reflected signal of frequency 
$\omega_{\text {read }}=\omega_{1}+\Delta \omega$ was filtered by another FBG, amplified and measured using either a low-bandwidth or a high-bandwidth detector, depending on the specific experiment.

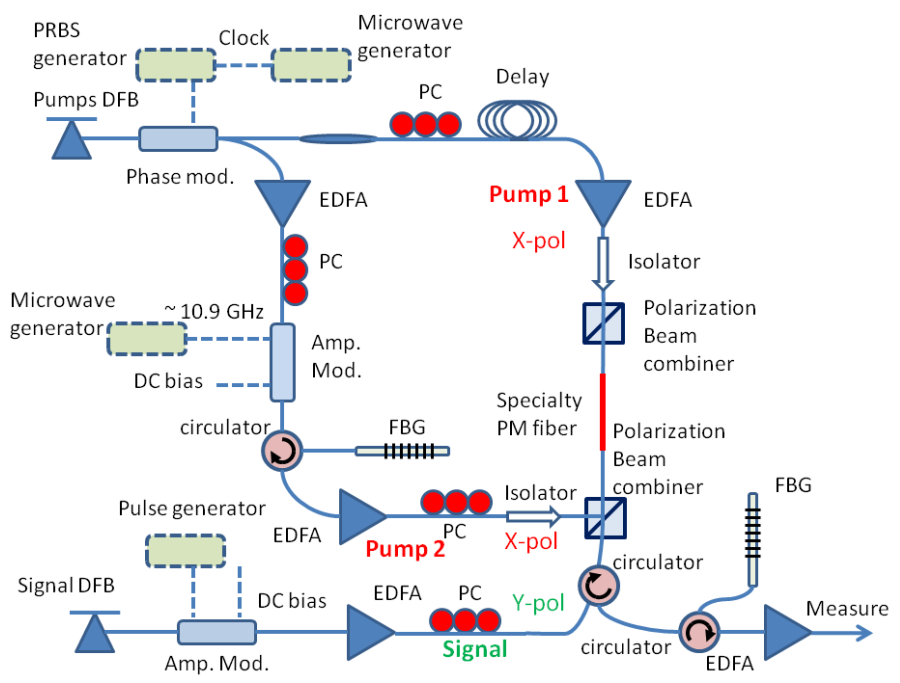

Fig. 2: Experimental setup for the generation and characterization of dynamic acoustic gratings, using SBS with phasemodulated pump waves. PC: polarization controller.

\section{RESULTS: VARIABLE DELAY USING STATIONARY AND LOCALIZED DYNAMIC GRATINGS}

The variable delay of isolated and periodic 1 ns-long readout pulses was demonstrated along a $100 \mathrm{~m}$ long FUT $\left(\Omega_{B}=\right.$ $2 \pi \cdot 10.87 \mathrm{GHz}, \Delta \omega=2 \pi \cdot 57 \mathrm{GHz}$ ). In this experiment, a PRBS of length $M=2^{10}-1$ was used in the modulation of both pumps, and a fiber delay imbalance was added to the path of pump 1 so that the $10^{\text {th }}$ correlation peak was scanned along the FUT. Figure 3 shows the relative delay of reflected pulses, using seven different symbol durations $T$. A variable delay of $770 \mathrm{~ns}$, or 770 times the temporal width of the readout pulses, is readily observed. (The right-most peak in Fig. 4 is due to a parasitic reflection at frequency $\omega_{\text {sig }}$ that was not filtered out.) Fine tuning of the variable delay on a sub-100 ps scale is shown in Fig. 4.

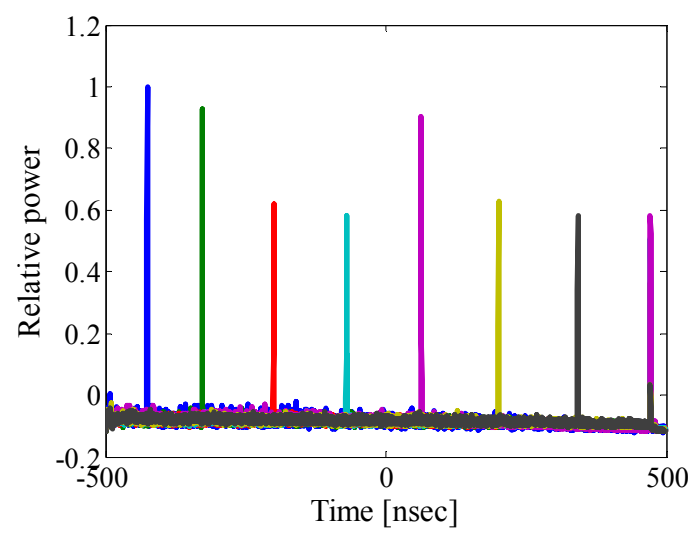

Fig. 3: Variable delay of reflected isolated and periodic readout pulses. The pulses were reflected from dynamic gratings, introduced by the $10^{\text {th }}$ correlation peak of phase-modulated pump waves. The PRBS modulation clock rates $1 / T$ were (left to right): $1.120 \mathrm{GHz}, 1.108 \mathrm{GHz}, 1.093 \mathrm{GHz}, 1.078 \mathrm{GHz}, 1.063 \mathrm{GHz}, 1.048 \mathrm{GHz}, 1.033 \mathrm{GHz}$. (The right-most peak, which is common to all PRBS rates, is due to a parasitic reflection.) 


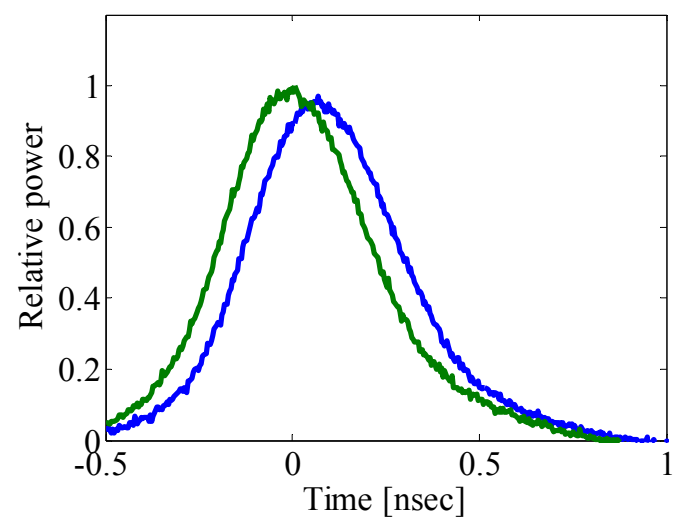

Fig. 4: Variable delay of reflected isolated and periodic readout pulses. The pulses were reflected from dynamic gratings, introduced by the $10^{\text {th }}$ correlation peak of phase-modulated pump waves. The difference between the PRBS modulation clock rate $1 / T$ in the two curves was $9 \mathrm{kHz}$, corresponding to a relative delay of $75 \mathrm{ps}$.

\section{RADIO-FREQUENCY-PHOTONIC FILTERS USING DYNAMIC GRATINGS}

Two-tap microwave photonic filters, based on the reflections from pairs of dynamic acoustic gratings, were demonstrated experimentally. The top panel of Fig. 5 shows the temporal reflection of isolated and periodic short readout pulses form pairs of dynamic gratings, having different separations. The grating pairs were generated via phase modulation of the pump waves with $M=2^{7}-1$ bits long PRBSs, with varying symbol durations $T$. Two localized reflections are apparent, separated by $\Delta t$ of $12.4 \mathrm{~ns}$ and $21 \mathrm{~ns}$. Since both reflections of the readout signal are phaseconjugated [1], it is expected that the two delayed replicas of the readout signal emerge out of the DUT with equal phases [1]. The frequency response of the two reflections combined is therefore equivalent to that of a two-tap filter with

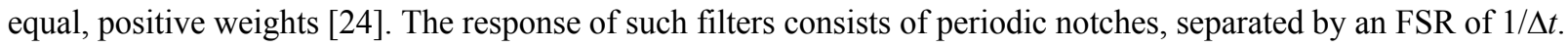
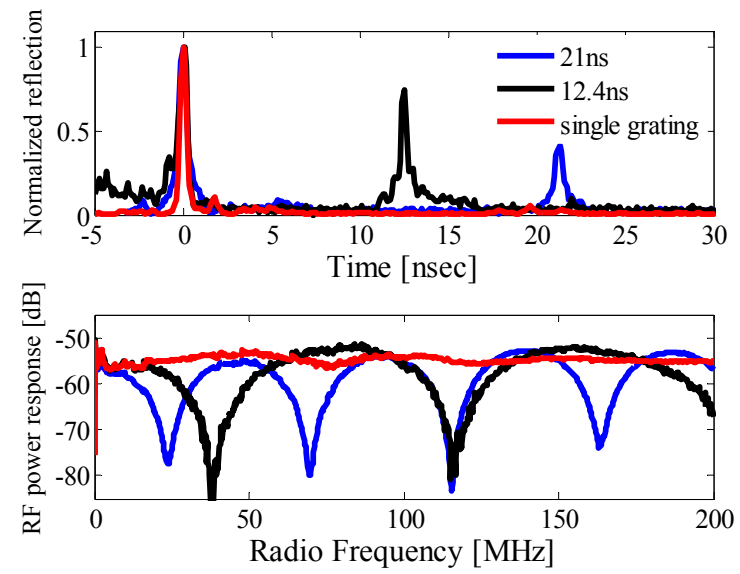

Fig. 5: Top - Temporal profile of the reflection of isolated, periodic readout pulses from a single dynamic gratin (red), and from pairs of gratings separated by $12.4 \mathrm{~ns}$ (black) and $21 \mathrm{~ns}$ (blue). Bottom - Vector network analyzer measurements of the power transfer function of the reflections from the single grating and grating pairs. The colors match those of the top panel.

The frequency response of the dynamic gratings-based filters was characterized using a vector network analyzer (VNA). An EOM in the readout signal path was driven by a swept sine-wave from the VNA output port, and the detected reflection was analyzed using the input port of the instrument. Figure 5 (bottom) shows the relative transfer function of the reflected signal power, as a function of the offset of the readout frequency from $\omega_{\text {sig }}$ of maximum reflectivity. 
Periodic spectral notches with the expected FSRs are apparent in the reflections from grating pairs. The FSR can be increased to $\mathrm{GHz}$ range with the use of shorter phase modulation sequences, which were unavailable to us at the time of the experiment.

\section{CONCLUDING REMARKS}

In this work, we have introduced and demonstrated a novel technique for the generation of localized and stationary acoustic gratings through SBS. The technique relies on the phase modulation of two optical pump waves by a common, high rate PRBS. The effective generation of the acoustic gratings is confined to discrete correlation peaks. The dynamic acoustic gratings serve as 'movable mirrors', that can be scanned along an FUT and provide a variable group delay of reflected readout pulses. The length of the gratings is governed by the short duration of the PRBS symbols, whereas the variable delay range is independently determined by the length of the sequence. The technique is therefore free, at least in principle, of delay-times-bandwidth product limitations. The wavelength conversion that accompanies the delay is minimal: an offset by $\Omega_{B}$ ). The delay of 1-ns-long readout pulses by as many as $770 \mathrm{~ns}$ was demonstrated experimentally. In addition, RF-photonic filters of variable FSR were demonstrated based on the combined reflections from a pair of localized acoustic gratings.

As mentioned as section 2 above, the expectation value of the acoustic grating magnitude outside the correlation peaks is zero. Nevertheless, the off-peak gratings have a non-zero standard deviation and a temporally varying magnitude (see the simulation results of Fig. 1, for example). Unfortunately, we have found through both analysis and simulations that the accumulative effect of scattering noise from residual gratings spanning the entire length of the FUT is severely restricting the optical signal to noise ratio (OSNR) of reflected, delayed waveforms:

$$
\operatorname{OSNR} \approx \frac{2 \tau}{T_{D}}
$$

where $T_{D}$ is the variable delay range. The OSNR was found to be largely independent of the PRBS symbol duration $T$. In the processing of optical communication data, the required OSNR should be at least on the order of 20-30. We therefore conclude that PRBS-driven dynamic gratings cannot delay an incoming data sequence with sufficient OSNR, unless the extent of delay is restricted to a fraction of the acoustic lifetime $\tau$, on the order of $1 \mathrm{~ns}$ or less. On-going work is focusing on more elaborate coding schemes in attempt to reduce the residual scattering noise. It remains that the technique offers an unprecedented flexibility for applications in which signal averaging is possible, such as the delay of repetitive signals. Ongoing work is being dedicated to the application of the proposed technique for the distributed sensing of temperature and strain over standard as well as PM fibers.

\section{REFERENCES}

[1] Boyd, R. W., [Nonlinear Optics], third edition, Academic Press (2008).

[2] Song, K. Y., Zou, W., He, Z., and Hotate, K., "All-optical dynamic grating generation based on Brillouin scattering in polarization-maintaining fiber," Opt. Lett. 33, 926-928 (2008).

[3] Dong, Y., Bao, X., and Chen, L., "Distributed temperature sensing based on birefringence effect on transient Brillouin grating in a polarization-maintaining photonic crystal fiber," Opt. Lett. 34, 2590-2592 (2009).

[4] Dong, Y., Chen, L., and Bao, X., "Truly distributed birefringence measurement of polarization- maintaining fibers based on transient Brillouin grating," Opt. Lett. 35,193-195 (2010).

[5] Zou, W., He, Z., and Hotate, K., "Complete discrimination of strain and temperature using Brillouin frequency shift and birefringence in a polarization-maintaining fiber," Opt. Express 17, 1248-1255 (2009).

[6] Zou, W., He, Z., Song, K. Y., and Hotate, K., "Correlation-based distributed measurement of a dynamic grating spectrum generated in stimulated Brillouin scattering in a polarization-maintaining optical fiber," Opt. Lett. 34, 1126-1128 (2009).

[7] Song, K. Y., Lee, K., and Lee, S. B., "Tunable optical time delays based on Brillouin dynamic grating in optical fibers," Opt. Express 17, 10344-10349 (2009). 
[8] Dong, Y., Chen, L., and Bao, X., "High-spatial-resolution simultaneous strain and temperature sensor using Brillouin scattering and birefringence in a polarization-maintaining fibre," IEEE Photonic. Technol. Lett. 22, 1364-1366 (2010).

[9] Song, K. Y., Chin, S., Primerov N., and Thevenaz, L., "Time-domain distributed fiber sensor with $1 \mathrm{~cm}$ spatial resolution based on Brillouin dynamic grating," J. Lightwave Technol. 28, 2062-2067 (2010).

[10] Song K. Y., and Yoon, H. J., "High-resolution Brillouin optical time domain analysis based on Brillouin dynamic grating," Opt. Lett. 35, 52-54 (2010).

[11] Song K. Y., and Yoon, H. J., "Observation of narrowband intrinsic spectra of Brillouin dynamic gratings," Opt. Lett. 17, 2958-2960 (2010).

[12] Dong, Y., Chen, L., and Bao, X., "Characterization of the Brillouin grating spectra in a polarization-maintaining fiber," Opt. Express 18, 18960-18967 (2010).

[13] Chin, S., Primerov, N., and Thevenaz, L., "Sub-Centimeter Spatial Resolution in Distributed Fiber Sensing Based on Dynamic Brillouin Grating in Optical Fibers," IEEE Sensors Journal 12, 189-194 (2012).

[14] Chin, S., Primerov, N., and Thevenaz, L., "Photonic delay line for broadband optical signals, based on dynamic grating reflectors in fibers," 2010 36th European Conference and Exhibition on Optical Communication (ECOC 2010), Torino, Italy, (2010).

[15] Thevenaz, L., "Slow and fast light in optical fibers," Nature Photonics 2, 474-481 (2008).

[16]Zadok, A., Eyal, A., and Tur, M., "Stimulated Brillouin scattering slow light in optical fibers," Appl. Opt. 50, E38-E49 (2011).

[17]Zhu, Z., Gauthier, D. J., Okawachi, Y., Sharping, J. E., Gaeta, A. L., Boyd, R. W., and Willner, A. E., "Numerical study of all-optical slow light delays via stimulated Brillouin scattering in an optical fiber," J. of Opt. Soc. Am. B 22, 2378-2384 (2005).

[18] Khurgin, J. B., "Performance limits of delay lines based on optical amplifiers," Opt. Lett. 31, 948-950 (2006).

[19] Boyd, R. W., and Narum, P., "Slow- and fast-light: fundamental limitations." J. Modern Optics 54, 2403-2411 (2007).

[20] Hotate, K., and Hasegawa, T., "Measurement of Brillouin gain spectrum distribution along an optical fiber using a correlation-based technique - proposal, experiment and simulation," IEICE Trans. Electron. E83-C, 405-412 (2000).

[21] Hotate, K., and Tanaka, M., "Distributed fiber Brillouin strain Sensing with 1-cm spatial resolution by correlation-based continuous-wave technique," IEEE Photon. Technol. Lett. 14, 179-181 (2002).

[22] Santagiustina, M., and Ursini, L., "Localized Dynamic Brillouin Gratings Permanently Induced by Chaotic Signals," in Signal Processing in Photonic Communications, OSA Technical Digest (CD) (Optical Society of America, 2011), paper JTuB6.

[23] Beugnot, J.-C., Tur, M., Foaleng Mafang S., and Thévenaz, L., "Distributed Brillouin sensing with sub-meter spatial resolution: modeling and processing," Opt. Express 19, 7381-7397 (2011).

[24] Capmany, J., Ortega, B., Pastor, D., and Sales, S., "Discrete-time optical processing of microwave signals", J. Lightw. Technol. 23, 702-723 (2005). 